\title{
Requerimientos de usuarios de la edificación traducidos en una metodología de apoyo a la decisión
}

\section{User requirements in building translated in a methodology of decision support}

$\underline{\text { C. Valderrama-Ulloa }}^{(*)(*)}$, J. R. Puiggali ${ }^{(* *)}$

\begin{abstract}
RESUMEN
Diferentes actores del sector de la edificación han desarrollado diversos métodos que evalúan la calidad de sus edificios en respuesta a problemáticas medioambientales. Métodos desarrollados en un contexto específico, en este último tiempo han alcanzado una gran popularidad, pero al mismo tiempo ambigüedad en su medición, confusión en el objetivo que persiguen, olvidando el contexto en los que han sido concebidos. En este trabajo se desarrolla una metodología de apoyo a la decisión que evalúa el comportamiento de edificios terciarios en fase de explotación desde el punto de vista energéticomedioambiental y satisfacción de sus usuarios, con el fin de decidir entre una rehabilitación o la demolición. El caso de estudio es un campus universitario en Francia, el cual reúne distintos usos y usuarios, y por lo tanto distintos requerimientos de calidad y de confort. Los indicadores que conforman la metodología consideran esta complejidad entregando sus resultados en un único perfil de evaluación, permitiendo ser aplicados en distintos contextos.
\end{abstract}

Palabras clave: Indicadores; certificación medioambiental; método multicriterio; sector terciario.

\section{ABSTRACT}

Different actors of building sector have developed various methods that assess the quality of its buildings in response to environmental issues. The methods developed in a specific context, have achieved a great popularity in recent years, but also an ambiguity in its measurement, a confusion in the objective pursued, forgetting the context in which they are intended. This paper develops a methodology of decision support, which evaluates the performance of existing buildings from the point of view of energy and user satisfaction, to decide between rehabilitation or demolition. The case of study is an university campus, which brings together different uses and users, and therefore different quality and comfort requirements. The indicators in the methodology consider this complexity, delivering its results in a single evaluation profile and allowing to be applied in different contexts.

Keywords: Indicators; environmental certification; multi-criteria methods; service sector.

(*) Pontificia Universidad Católica de Chile, Santiago (Chile)

(**) Université de Bordeaux, Talence (Francia)

Persona de contacto/Corresponding Author: crvalder@uc.cl (C. Valderrama-Ulloa)

Cómo citar este artículo/Citation: Valderrama-Ulloa, C., Puiggali, J. R. (2014). Requerimientos de usuarios de la edificación traducidos en una metodología de apoyo a la decisión. Informes de la Construcción, 66(534): eo22, doi: http://dx.doi.org/10.3989/ ic.12.106.

Licencia/License: Salvo indicación contraria, todos los contenidos de la edición electrónica de Informes de la Construcción se distribuyen bajo una licencia de uso y distribución Creative Commons Reconocimiento no Comercial 3.o. España (cc-by-nc). 


\section{INTRODUCCIÓN}

El desarrollo de sistemas de evaluación medioambiental de edificaciones ha sido motivado por el aumento de la sensibilización pública sobre los temas medioambientales, destacando la necesidad de las empresas constructoras y mandantes a tener herramientas para estimar sus impactos sobre el medioambiente, incluidas dentro de las estrategias de gestión de riesgos de las empresas (1).

Los sistemas de evaluación en las edificaciones se pueden utilizar para evaluar la calidad ambiental o energética ya sea en un contexto local o regional, durante las fases de diseño, construcción o explotación y para su uso en empresas, viviendas o edificios terciarios. En estas metodologías podemos encontrar sistemas con puntuaciones (como LEED), notas (como CASBEE), niveles de comportamiento (como HQE), entre otras opciones. Por otra parte estos sistemas pueden evaluar cada problemática de los edificios (uso del agua, uso de la energía, confort, desechos, impactos ambientales, entre otros) por separado o integrándolas en un sólo criterio, lo que facilita su lectura.

Los primeros indicadores de sostenibilidad fueron propuestos alrededor del mundo desde la década de los '9o (2) o (3) y hoy podemos encontrar más de 800 entradas registradas en la base de datos del compendio de la iniciativas de indicadores del desarrollo sostenible (4). Este auge refleja la gran popularidad que han alcanzado estos indicadores, lo cual desafortunadamente se ha visto acompañada por un aumento en la ambigüedad de su evaluación y confusión en el objetivo de caracterización de cada uno y por lo tanto en sus métodos y medidas de evaluación (5).

Por otro lado, como describen Macías y García Navarro (6), las certificaciones presentan puntuaciones desarrolladas para una región determinada, ofreciendo simplicidad y facilidad en su aplicación. Sin embargo cuando el edificio cambia de lugar, los resultados pueden dejar de ser objetivos. Y por otra parte la visión de evaluación casi siempre se centra en el edificio como un elemento aislado, con su entorno inmediato en el mejor de los casos, pero ¿qué pasa con los usuarios, fin último de la cadena de valor?

El método de evaluación que se describe, no pretende ser una nueva certificación, sino una metodología que intenta homogeneizar indicadores recurrentes y que son en la mayoría de los casos considerados como los más importantes en las distintas certificaciones revisadas (CASBEE, LEED, HQE, BREEAM, DGNB y LBC). A través de la presente metodología podemos evaluar diferentes indicadores en un perfil homogéneo de evaluación, utilizando un método de análisis multicriterio podemos integrarlos en sólo valor de evaluación.

El objetivo de esta metodología es evaluar edificios terciarios en fase de explotación, en donde la literatura nos indica que es un área que aún no está plenamente desarrollada. Por ejemplo, Ding (7) señala que el esfuerzo en esta fase todavía no es suficiente, pues los principales métodos de evaluación medioambiental de los edificios están aplicados predominantemente a las nuevas construcciones. O como Crawley y Aho (8) justifican: la razón del por qué estas evaluaciones se utilizan principalmente en la fase de la construcción es por el coste, pues el trabajo a realizar en la fase de explotación puede ser demasiado extenso y requerir una inversión importante de tiempo.
Y por otro lado como la construcción y demolición son fases de la vida de un edificio que consumen una elevada cantidad de energía, son las fases que más les interesan a las empresas constructoras y mandantes, pero desde el punto de vista energético y sostenible son fases pocas significativas si las comparamos con el uso del edificio (fase de explotación) (9) (10).

Como caso de estudio se ha elegido dentro del sector terciario: un campus universitario. Esto suma complejidad a la evaluación, pues los campus universitarios tienen la particularidad de estar conformados por un conjunto de edificios, con usos y usuarios muy heterogéneos, lo que dificulta la utilización de una evaluación única y homogénea para todo el conjunto.

El campus universitario evaluado es el Campus Principal «Ciencias y Tecnologías» de la Universidad de Burdeos en Francia. Este se extiende en una superficie de 120 hectáreas de terreno, con $165.000 \mathrm{~m}^{2}$ construidos, representando 45 edificios ligados a la investigación, a la docencia o a la administración (11). Este contexto dificulta la lectura de los datos a evaluar y por lo tanto uno de los objetivos de este estudio es homogenizar estos datos, considerando la apreciación de los usuarios en la evaluación final.

Esta metodología nos permite evaluar el comportamiento de estos tipos de edificios desde el punto de vista energético-medioambiental y la satisfacción de sus usuarios, información preliminar que permitirá al gestor de la universidad decidir entre: continuar su explotación con una leve mejora en su gestión, rehabilitar mejorando uno o algunos de los criterios que conformarán los indicadores o definitivamente demoler.

\section{FASES DEL ESTUDIO}

A continuación se describen las etapas del estudio:

- Presentación de la metodología:

En este capítulo se describirán los principales elementos que conforman la metodología de evaluación: el perfil de los usuarios, la relación entre usuarios - indicadores y pilares del desarrollo sostenible, así como los diferentes modelos adoptados.

- Construcción de la metodología:

A partir de la definición de un indicador el objetivo de esta parte es construir la línea base de los indicadores que conforman la metodología de evaluación. Esta construcción se realiza a partir de las temáticas privilegiadas en diferentes certificaciones internacionales, reglamentaciones nacionales o locales (francesas o europeas) y del análisis de las unidades, características de medidas y tipos de intervalos adoptados en estas referencias.

\section{- Aplicación de la metodología:}

Finalmente, a partir de los elementos definidos en la etapa anterior, se presenta los resultados de la evaluación de tres tipos de edificios (docencia, investigación y administración) del Campus Universitario «Ciencias y Tecnologías» de la Universidad de Burdeos en Francia. 


\subsection{Presentación de la metodología}

La metodología consiste en la construcción de una matriz de criterios que se traducirá en requerimientos de gestores o de usuarios de edificios terciarios concernientes a las grandes temáticas del desarrollo sostenible. Esta metodología considera actividades específicas desarrolladas en los campus universitarios, es decir aplicable a edificios de oficinas, laboratorios o edificios de docencia.

A partir de un estudio anterior (12) que realizó una detallada comparación entre las distintas temáticas evaluadas en certificaciones internacionales, reglamentaciones, normativas o trabajos de investigación, en este estudio se han elegido los indicadores más representativos de ese trabajo: consumo energético, la utilización de energías renovables, las emisiones de dióxido de carbono $\left(\mathrm{CO}_{2}\right)$, confort acústico, visual, térmico y olfativo. Con la función de proporcionar escenarios de decisión en dichas temáticas, estos indicadores se han agrupado en los tres pilares del desarrollo sostenible: el consumo de recursos al pilar económico, los impactos medioambientales al pilar medioambiental y la calidad del ambiente interior al pilar social (Figura 1).

Por otra parte cada uno de los diferentes actores de un campus universitario (gestor, tipo de edificios y usuarios), se han asociado a uno de los tres pilares del desarrollo sostenible, dando nacimiento a los tres escenario de decisión que se utilizarán en la metodología.

\subsubsection{Los diferentes elementos de la metodología}

En la universidad de Burdeos intervienen varios elementos durante su fase de explotación: los usuarios (estudiantes, profesores-investigadores, administrativos), la dirección «gestor» (quien toma las decisiones) y los edificios a evaluar (en la universidad principalmente del tipo administrativo, de docencia y de investigación).

La evaluación de sus edificios no es una tarea fácil, pues existe una gran variabilidad entre sus años de construcción (años '60 y esta última década), entre sus superficies construidas (de $900 \mathrm{~m}^{2}$ a $11.069 \mathrm{~m}^{2}$ útiles), entre su tasa de ocupación (vacaciones diferidas, horarios distintos), así como entre su presencia a nivel de superficie (46\% del total de la superficie construida son edificios de investigación, $34 \%$ de docencia, $19 \%$ de administración y $1 \%$ de otro tipo de uso) (11).

Las relaciones entre usos y usuarios casi nunca es evidente: por un lado los usuarios utilizan el edificio para desarrollar sus actividades, mientras que el gestor debe velar por el buen funcionamiento de los edificios. Esto con el fin de que se desarrollen satisfactoriamente estas actividades, buscando un balance adecuado entre la satisfacción del confort de los usuarios y una gestión financiera.

Con la finalidad de simplificar la problemática relacionada a la multiplicidad de actores y escenarios de evaluación (Figura 1), hemos unido la visión de ambos a través de una matriz de jerarquización. Esta herramienta permite identificar el nivel de apreciación que cada actor le concede a cada indicador y a cada escenario definido.

Para establecer el nivel de apreciación que los usuarios (estudiantes, profesores-investigadores, administrativos) les entregan a los indicadores y a los escenarios, se realizó una encuesta en la Universidad de Bordeaux-campus Ciencias y Tecnologías, durante el mes de marzo 2012. Un total de 800 personas vía on-line de 11.500 respondieron la encuesta, resultados que nos permitieron construir la matriz de jerarquización. La encuesta consistió en pedir a los usuarios ordenar los 7 indicadores (consumo energético, energías renovables, emisiones de $\mathrm{CO}_{2}$, confort acústico, visual, térmico y olfativo) y los 3 escenarios (económico, social y medioambiental) del menos al más importante.

La construcción de la matriz utilizó el Proceso de Análisis Jerárquico $(A H P)$ (13), cuyo objetivo es calcular la prioridad (definida como $w_{i}$ en el modelo) de cada elemento (los 7 indicadores y los tres escenarios). El uso de la matriz $A H P$ se caracteriza por la facilidad y sencillez en su uso, ya que permite evaluar un gran número de criterios entregando una única solución óptima (14).

Finalmente, para determinar el valor de cada indicador y de cada escenario en la evaluación del edificio, se ha utilizado y adaptado la base metodológica del método de optimización multicriterio, él que se describe a continuación.

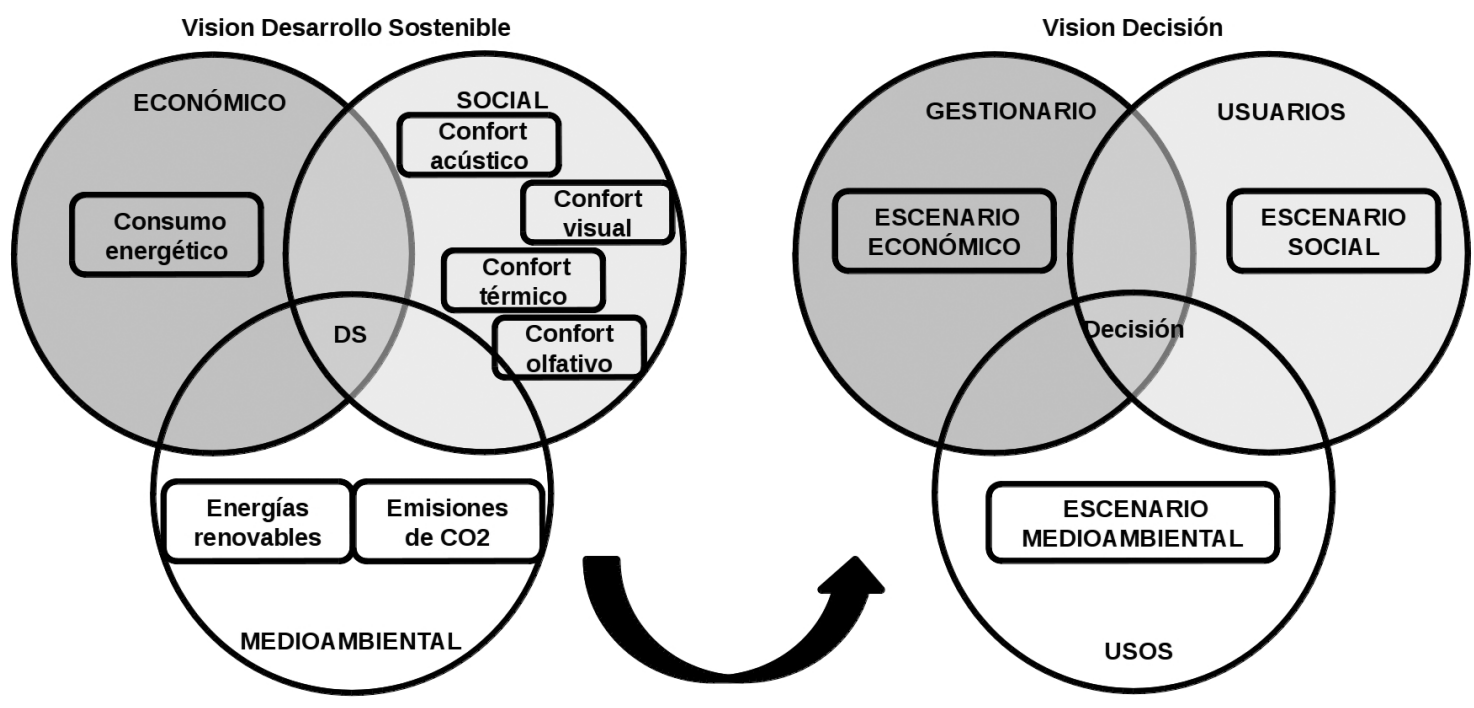

Figura 1. Los indicadores con los pilares y los escenarios con los actores. 


\subsubsection{El método de optimización multicriterios}

La optimización es un enjambre matemático que busca analizar y resolver analíticamente o numéricamente el problema de determinar el mejor elemento de un conjunto.

El método de optimización utilizado está formado por 3 modelos de aplicación consecutiva: el de interpretación, que tiene por objetivo transformar los indicadores en valores adimensionales a través de una función llamada «de aceptabilidad»; el de agregación que tiene por objetivo unir las variables de interpretación con los escenarios de decisión (IGA) y los escenarios de decisión en un sólo valor (IFE) a través de la función de agregación; y finalmente, el modelo de decisión, que permite determinar en una fase preliminar y técnica si el edificio es rehabilitado, demolido o si se continúa con su explotación con una leve modificación. La Figura 2 muestra en forma sintética estos tres modelos.

\subsubsection{El modelo de Interpretación}

Este modelo nos permite transformar nuestros indicadores en funciones de optimización (minimizar [1], delimitar [2] o maximizar [3]), que transforman a través de una función de aceptabilidad nuestro indicador en un valor numérico adimensional.

La función de aceptabilidad definida por Harrigton (15) y Derringer y Suich (16), es una función de optimización que es aplicable sólo para casos lineares y cuando existe sólo un tramo de medida. En nuestro caso de estudio hay 12 criterios de evaluación con distintos niveles de medición, por lo que para volverlos comparables y analizables se ha utilizado la función de aceptabilidad adaptada por Sebastian et al. (17) y Wagner y Trautmann (18).

Esta función evalúa el porcentaje de cumplimiento (o grado de aceptación) de cada criterio y asegura la transformación de todas las funciones objetivo en una escala de aceptabilidad con valores entre 0,01-0,99.

Las siguientes ecuaciones serán utilizadas para normalizar los criterios en función del objetivo deseado, por ejemplo al buscar minimizar el consumo energético [1], delimitar la temperatura interior [2] o maximizar la satisfacción olfativa [3].

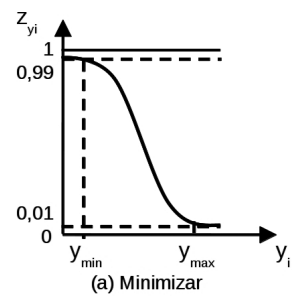

$$
z_{y i}=\exp \left(-\exp \left(\beta+\alpha \cdot y_{i}\right)\right)
$$

$\operatorname{con}\left\{\alpha=\frac{\ln \left(\frac{\ln (0,01)}{\ln (0,99)}\right)}{y_{\max }-y_{\min }}, \beta=\ln (-\ln (0,99))-\alpha \cdot y_{\text {min }}\right.$

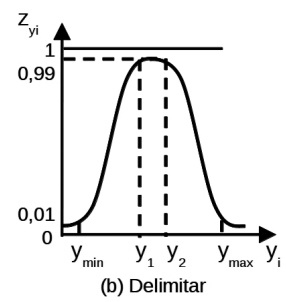

$$
\begin{aligned}
& z_{y i}=\exp \left(-\left(\frac{2 \cdot y_{i}-(U+L)}{U-L}\right)^{n}\right) \\
& \operatorname{con} n=\frac{\ln (-\ln (0,99))}{\ln \left(\mid \frac{2 \cdot y_{i}-U-L}{U-L}\right)}, U=\frac{y_{\max }+y_{2}}{2} \text { y } L=\frac{y_{\text {min }}+y_{1}}{2}
\end{aligned}
$$

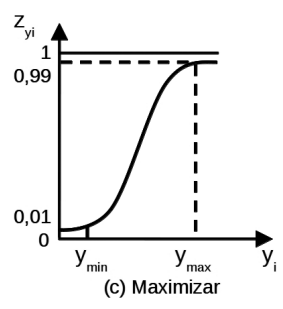

$z_{y i}=\exp \left(-\exp \left(\beta+\alpha \cdot y_{i}\right)\right)$

$\operatorname{con}\left\{\alpha=\frac{\ln \left(\frac{\ln (0,01)}{\ln (0,99)}\right)}{y_{\text {max }}-y_{\text {min }}}, \beta=\ln (-\ln (0,99))-\alpha \cdot y_{\text {max }}\right.$

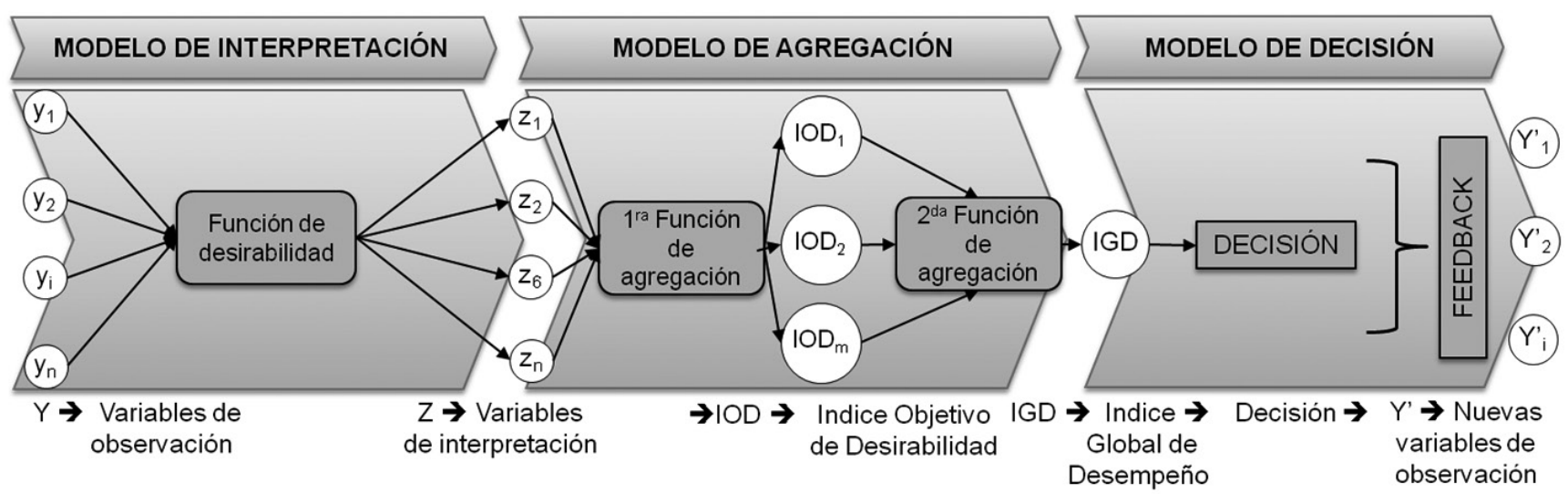

Figura 2. Los tres modelos de la metodología de evaluación. 


\subsubsection{El modelo de Agregación}

El segundo paso de la metodología es el modelo de «Agregación», como su nombre lo indica, nos permite agregar a través de una Función de Agregación $(F A)$ las evaluaciones individuales de cada indicador en cada escenario y de cada escenario en un evaluación final, considerando el nivel de apreciación de los usuarios.

Para realizar esta combinación hemos utilizado, en dos ciclos, la función de agregación que Scott y Antonsson (19) proponen [4]. La primera $F A$ agrega los valores $Z\left(y_{i}\right)$ de los indicadores de acuerdo a la preferencia $\left(w_{i}\right)$ obteniendo el «Índice Global de Aceptabilidad - IGA» para cada escenario. La segunda $F A$ agrega el valor de los $I G A$ con sus respectivas preferencias $\left(w_{i}\right)$, obteniendo el «Índice Final de Evaluación - IFE».

$F . A .=\sqrt[s]{\frac{w_{i} \times z\left(y_{i}\right)^{s}+\ldots+w_{n} \times z\left(y_{n}\right)^{s}}{w_{i}+\ldots+w_{n}}}$, donde:

- $w_{i}=$ preferencia de cada indicador o de cada escenario

$-z_{y i}=$ variable de interpretación de cada indicador o de cada escenario

$-\mathrm{s}=$ nivel de compromiso $[-\infty, 0,1+\infty]$

El nivel de compromiso (s)

El valor (s) representa el nivel de compromiso que se le otorga al valor de evaluación, es decir determina si se considera un escenario pesimista o uno optimista. La elección este valor determina el método de cálculo a utilizar para la agregación. Aquí se presentan las 4 más utilizadas (19):

1. Valor mínimo $(s \rightarrow-\infty): I G A$ toma el valor mínimo [5] de todos los valores de interpretación $\left(z_{v i}\right)$. Con ello nos aseguramos que en el valor final de evaluación (IFE) le damos una atención especial a los valores mínimos, como una manera de buscar la mejora del peor comportamiento. Una desventaja a señalar es que los otros valores $z_{v i}$ y el nivel de preferencia $\left(w_{i}\right)$ no son considerados.

$$
I G A=\min x \in\{1, X\} \text { de } Z(y)
$$

2. Ponderación de nulidad $(s \rightarrow-0)$ : la función genérica del $I G A[6]$ es igual a la media geométrica ponderada de todos los valores de interpretación asociados a cada indicador o a cada escenario. Este tipo de función de agregación es útil cuando existe un valor mínimo, como forma de alertar al gestor para que las medidas que tome tengan como objetivo mejorar el peor valor.

$$
I G A=w_{1} \cdot Z\left(y_{1}\right)^{w_{i}} \times \ldots \times w_{N} \cdot Z\left(y_{n}\right)^{w_{N}}
$$

3. Ponderación unitaria $(s \rightarrow 1)$ : la función genérica del $I G A$ [7] es igual a la media aritmética ponderada de todos los valores de interpretación asociados a cada criterio. Aquí el objetivo buscado es compensar el peor valor por el más fuerte, es decir se acentúa el mejor comportamiento.

$$
I G A=w_{1} \cdot Z\left(y_{1}\right)+\ldots+w_{N} \cdot Z\left(y_{N}\right)
$$

4. Valor máximo $(s \rightarrow+\infty): I G A$ toma el valor máximo [8] de todos los valores de interpretación $\left(z_{v i}\right)$. Con ello nos aseguramos que en el valor final de evaluación (IFE) mostremos el mejor comportamiento. Al igual que en el valor mínimo se pierde la información de los otros valores $z_{v i} y$ el nivel de preferencia.

$$
I G A=\max x \in\{1, X\} \text { de } Z(y)
$$

\subsubsection{El Modelo de Decisión}

Finalmente, a partir del modelo de Interpretación (transformación de los indicadores a valores matemáticos), pasando por el modelo de Agregación (unión de cada indicador en los escenarios y de cada escenario en el $I F E$ ), se llega al modelo de decisión: entre continuar con la explotación, definir una rehabilitación o demoler.

En función de los valores encontrados como IFE [0,01 0,99], del muy malo a muy bueno, se le muestran al gestor el nivel de cumplimiento de los indicadores, de los escenarios establecidos y del comportamiento del edificio. Esta evaluación técnica preliminar considera la importancia que le entregan los usuarios de un campus universitario a diferentes criterios energéticos-medioambientales.

\subsection{Construcción de la metodología}

\subsubsection{Los indicadores}

Un indicador es un descriptor, una medida (cuantitativa o cualitativa) que describe un fenómeno. El usuario elige un indicador para que él lo retroalimente sobre el estado global de un problema. Además posee una mutua dependencia con el contexto de utilización, ya que si este es «sacado» del contexto en el que fue creado, el indicador no tendrá ni el mismo sentido ni el mismo valor. Es por esto que no existen buenos indicadores por sí solos, sino más bien indicadores apropiados a un contexto particular.

En nuestra metodología el objetivo del indicador es evaluar la calidad de una variable (los 12 criterios) o un conjunto de variables (escenarios) en función al objetivo fijado por el decididor (minimizar consumos o maximizar satisfacción), entregando así una visión sintética y simple del sistema (comportamiento de edificios terciarios en fase de explotación), facilitando la gestión y la toma de decisión (rehabilitacióndemolición).

Particularmente, en un proyecto de construcción las funciones de un indicador pueden ser de cuatro tipos:

- De diagnóstico: constituyen en un momento dado un «termómetro» de problemas locales y de su medioambiente.

- De control: muestran las evoluciones del proyecto en función a la situación inicial o de los objetivos de partida, permiten medir el o los impactos del proyecto en marcha, así como de prever los potenciales impactos futuros.

- De comunicación: para sus usuarios, sus creadores y todos los actores involucrados.

- De acompañamiento a la decisión: permiten evaluar la eficacia de las políticas puestas en marcha y reorientar estrategias. 
Finalmente, en la metodología la manera de «evaluar» el cumplimiento del objetivo fijado por el gestor será a través de intervalos, los cuales nos permitirán determinar el cumplimiento del indicador.

\subsubsection{Comparación de certificaciones y referencias internacionales}

Para definir los indicadores e intervalos de medidas que conforman esta presente metodología, la primera etapa consistió en una detallada revisión de las temáticas (12) (gestión, sitio, medioambiente interior, consumo de recursos, impactos medioambientales, dimensión socio económica e innovación y diseño) que conforman las principales certificaciones internacionales.

La Tabla 1, resume las principales temáticas evaluadas y encontradas en las distintas certificaciones (marcadas con un $\sqrt{ }$ ), comparación que nos permitió definir los principales indicadores que utilizaremos en esta metodología.

Las versiones de las certificaciones utilizadas fueron:

- Leadership in Energy and Environment Design (LEED) Canada pour bâtiments existants: exploitation et entretien. Système d'évaluation. (2009).

- Comprehensive Assessment System for Built Environment Efficiency (CASBEE) for New Construction. Technical Manual v.1.o (2010).

- Haute Qualité Environnementale (HQE) Réferentiel Technique de Certification. Bâtiments Tertiaires en Exploitation (2009).
- Building Research Establishment Environmental Assessment Methodology (BREEAM) Bespoke International Assessor Manual, Issue 1.o (2008).

- German Sustainable Building Certificate (DGNB) International : Structure-Application-Criteria (2010).

- Living Building Challenge $\left(L B C^{T M}\right)$. A visionary path to a restorative future v2.o (2010).

Posteriormente se realizó una complementaria revisión de las diferentes definiciones, unidades, características de medidas y tipos de intervalos adoptados, para cada uno de los indicadores retenidos de la tabla anterior.

Esta revisión se realizó nuevamente en las certificaciones descritas y en reglamentaciones internacionales y nacionales (20), normativas europeas (21), como por ejemplo PASSIVHAUS (sello de calidad alemán que mide el comportamiento energético de los edificios), MINERGIE-P (sello de calidad suizo) o BBC-EFFINERGIE (sello de calidad francés), en algunos trabajos científicos (22), (23), (24), (25) y (26) y en la información de la Organización Mundial de la Salud (OMS) (27) y (28).

La Tabla 2, resume los indicadores, criterios e intervalos adaptados en esta metodología.

Como se observa en la Tabla 2 los intervalos pueden ser cerrados debido a que si se pasa de esos valores la evaluación se castiga entregando automáticamente el valor mínimo, de lo contrario al ser intervalos abiertos, se continua la evaluación sin castigar el valor, haciendo referencia a

Tabla 1. Comparación de las certificaciones y referencias internacionales.

\begin{tabular}{|l|c|c|c|c|c|c|l|}
\hline \multirow{2}{*}{ TEMÁTICAS } & \multicolumn{5}{c|}{ CERTIFICACIONES INTERNACIONALES } & \multicolumn{2}{c|}{ METODOLOGÍA } \\
\cline { 2 - 8 } & CASBEE & LEED & HQE & BREEAM & DGNB & LBC & \\
\hline Gestión & $\sqrt{ }$ & $\sqrt{ }$ & $\sqrt{ }$ & $\sqrt{ }$ & $\sqrt{ }$ & & Visión gestor \\
\hline Sitio & $\sqrt{ }$ & $\sqrt{ }$ & $\sqrt{ }$ & $\sqrt{ }$ & $\sqrt{ }$ & $\sqrt{ }$ & No se aplica - fase de explotación \\
\hline Ambiente interior & $\sqrt{ }$ & $\sqrt{ }$ & $\sqrt{ }$ & $\sqrt{ }$ & $\sqrt{ }$ & $\sqrt{ }$ & Confort: olfativo - visual - acústico - térmico \\
\hline Consumo de recursos & $\sqrt{ }$ & $\sqrt{ }$ & $\sqrt{ }$ & $\sqrt{ }$ & $\sqrt{ }$ & $\sqrt{ }$ & Consumo de energía final - Matriz energética \\
\hline Impactos medioambientales & $\sqrt{ }$ & & $\sqrt{ }$ & $\sqrt{ }$ & $\sqrt{ }$ & & Emisiones de $\mathrm{CO}_{2}$ \\
\hline Dimensión socio económica & $\sqrt{ }$ & & & & $\sqrt{ }$ & $\sqrt{ }$ & $\begin{array}{l}\text { Escenarios: Social - Medioambiental - Eco- } \\
\text { nómico }\end{array}$ \\
\hline Innovación y diseño & & $\sqrt{ }$ & & $\sqrt{ }$ & $\sqrt{ }$ & $\sqrt{ }$ & No en esta versión \\
\hline
\end{tabular}

Tabla 2. La definición de los diferentes indicadores retenidos en la metodología de evaluación.

\begin{tabular}{|c|c|c|c|}
\hline INDICADOR & CRITERIO & UNIDAD & $\begin{array}{c}\text { INTERVALO } \\
] \mathbf{y}_{\mathrm{o}}-\mathrm{y}_{1}[\end{array}$ \\
\hline Consumo Energético & Consumo de gas y electricidad & $\mathrm{kWh}_{\text {EnerǵaFinal }}$ anuales $/ \mathrm{m}^{2}$ & ] $50-300]$ \\
\hline Energías Renovables & Producción de energías renovables & $\mathrm{kWh}_{\mathrm{EF}}$ anuales $/ \mathrm{m}^{2}$ & {$[0-50[$} \\
\hline Emisiones de $\mathrm{CO}_{2}$ & Cantidad de emisiones de $\mathrm{CO} 2$ & $\mathrm{~kg} \cdot{ }_{\text {eq }}$ COanuales $/ \mathrm{m}^{2}$ & ] $5-145]$ \\
\hline Confort Olfativo & Satisfacción olfativa & $\%$ & {$[0-100]$} \\
\hline \multirow{3}{*}{ Confort Visual } & $\begin{array}{l}\text { Cantidad de vistas de la envolvente del } \\
\text { espacio evaluado }\end{array}$ & $\%$ & {$[0-50[$} \\
\hline & Distancia (máx) a las vistas & $\mathrm{m}$ & {$[0-5[$} \\
\hline & Cantidad de iluminación & Lux & ] $250-2500]$ \\
\hline Confort Acústico & Nivel de ruido & $\mathrm{dB}(\mathrm{A})$ & ] $32-50]$ \\
\hline \multirow{4}{*}{ Confort Térmico } & Temperatura interior & ${ }^{\circ} \mathrm{C}$ & ] $17-25[$ \\
\hline & Velocidad del aire interior & $\mathrm{m} / \mathrm{s}$ & ] $0,15-0,25[$ \\
\hline & Humedad relativa & $\%$ & ] $40-60[$ \\
\hline & Satisfacción térmica & $\%$ & {$[0-100]$} \\
\hline
\end{tabular}


las funciones de aceptabilidad presentadas anteriormente [1] [3].

\subsection{Aplicación de la metodología}

Para validar la metodología de evaluación se realizaron diferentes campañas de medidas en tres tipos de edificios, donde cada uno representa diferentes actividades realizadas en la universidad de Burdeos: administración (oficinas), docencia (salas de conferencias y salas de clases) e investigación (laboratorios).

La elección de estos edificios y de los diferentes recintos evaluados se basó en tres criterios:

- grado de representatividad de estos edificios y recintos en función al resto de los edificios del campus y de los recintos dentro del mismo edificio (superficie, distribución, orientación),

- ser edificios y recintos que poseen una alta tasa de ocupación y

- ser edificios que tuvieran un historial de medidas energéticas,

La descripción de los edificios se presenta a continuación (Figura 3, Figura 4 y Figura 5):

- Investigación (laboratorio de química molecular) - 1966 $11.069 \mathrm{~m}^{2}$ (superficie útil) - 6 pisos

Las medidas fueron realizadas en dos oficinas (de 2 a 3 personas, en el 1er piso), orientación sureste y suroeste y dos laboratorios (de 2 a 3 personas, en el 1er piso) con una orientación noreste y noroeste.
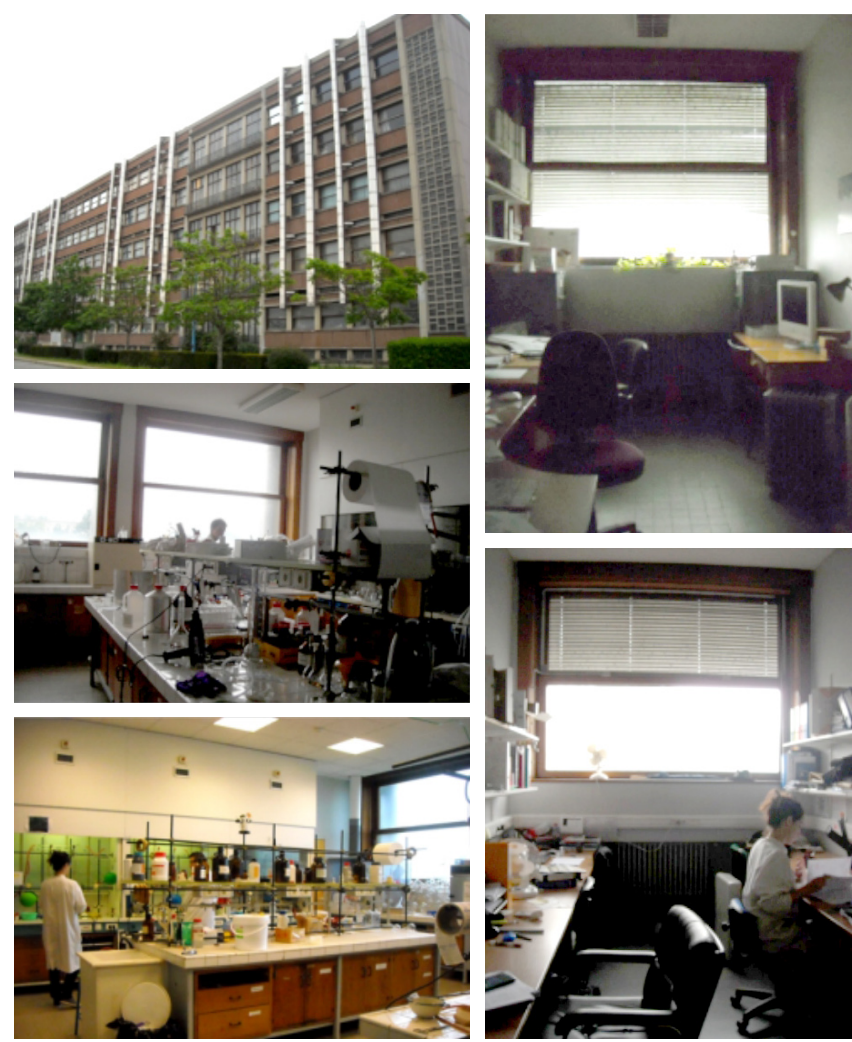

Figura 3. Fotografías y descripción de los locales evaluados en el edificio de investigación.
- Docencia - 1966

$2.300 \mathrm{~m}^{2}$ (superficie útil) - 4 pisos

Las medidas fueron realizadas en el anfiteatro (capacidad para 180 personas en dos niveles: 2 do y 3 er piso), orientación norte y sudoeste y en una sala de clases (con capacidad para 35 personas, en el zer piso), orientación sudeste.

- Administración (Comprado por la universidad en 1992) $7.230 \mathrm{~m}^{2}$ (superficie útil) - 3 pisos

Las medidas fueron realizadas en dos oficinas individuales en el 2do piso con orientaciones sudeste y noroeste.

Las medidas de los diferentes criterios $\left(y_{i}\right)$ para cada edificio fueron determinadas a través de:

- lectura de los consumos eléctricos anuales de cada edificio y una estimación del consumo de gas a partir del consumo anual de la universidad.

- campañas de medidas durante dos meses de invierno y dos meses de transición.

- entrevistas a los usuarios de los tres edificios.

El valor de interpretación $\left(z_{i}\right)$ de cada criterio se calculó a partir de la función objetivo $(F O)$ definida en las ecuaciones [1] [2] y [3].

Los diferentes valores $\left(y_{i}\right)$ obtenidos de la campaña de medida, el tipo de $F O$ utilizada en cada uno y los valores de las variables de interpretación para cada criterio $\left(z_{i}\right)$ se resumen en la Tabla 3 .
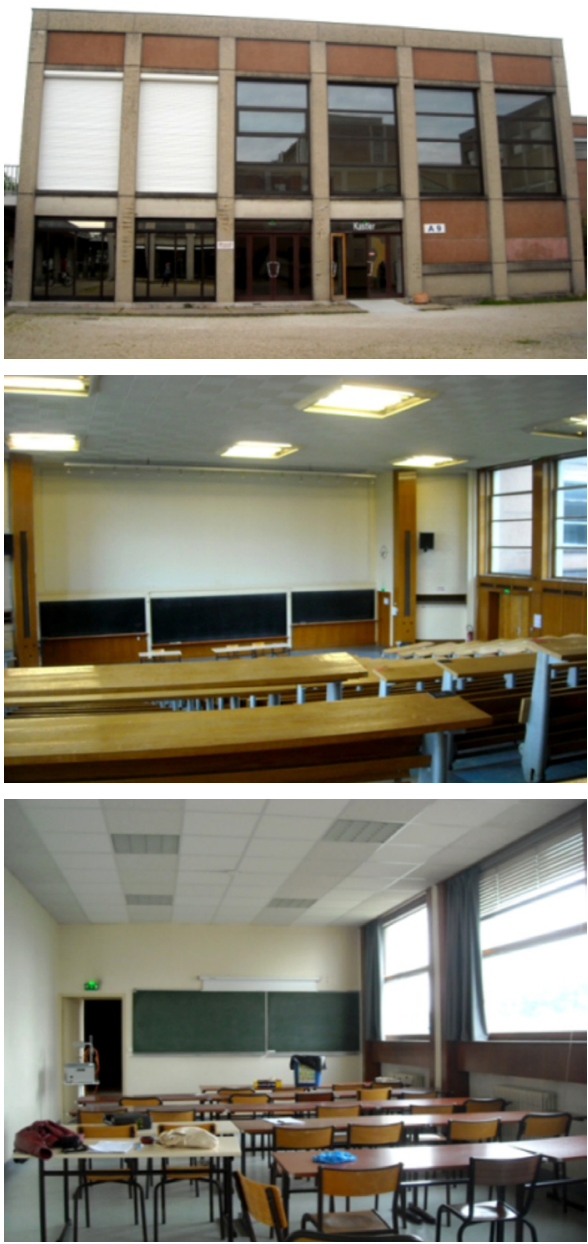

Figura 4. Fotografías y descripción de las salas evaluadas en el edificio de docencia 


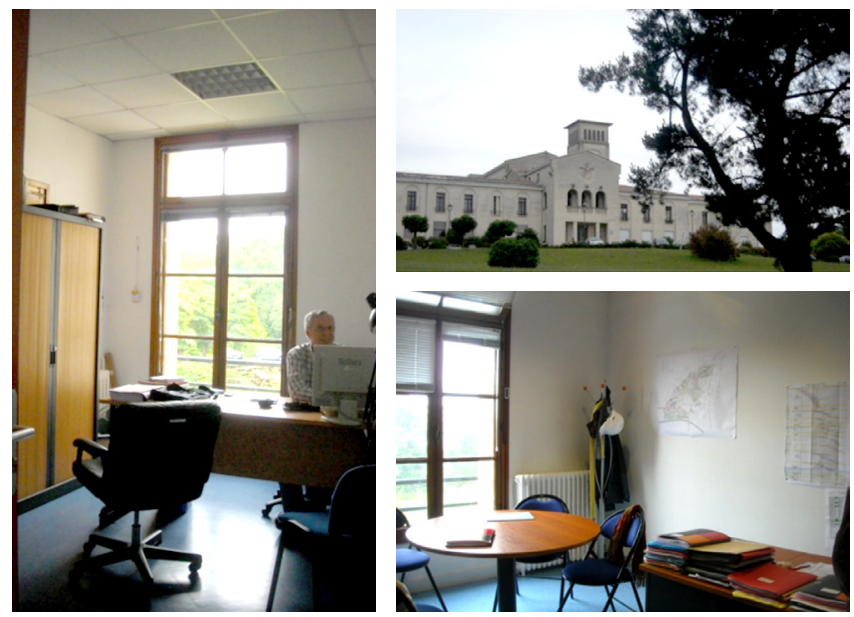

Figura 5. Fotografías y descripción de las oficinas evaluadas en el edificio de administración.

De manera ilustrativa y con el objetivo de alertar al decidor de que hay un comportamiento deficiente en el conjunto de criterios que conforman el indicador, en los resultados de este trabajo sólo se presentará el escenario pesimista, es decir el valor de cada indicador $\left(Z_{i}\right)$ será igual al peor valor del conjunto de criterios que lo conforman. Por ejemplo en el edificio de investigación el confort visual es igual a 0,01, elegido a partir del valor de la cantidad de iluminación $(0,01)$ por ser el peor.

Como podemos observar en el perfil de evaluación (representación gráfica de las variables de interpretación $Z_{i}$ ) de la Figura 6, los indicadores acústicos, olfativo y emisiones de $\mathrm{CO}_{2}$ son los indicadores con un cumplimiento casi excelente (en promedio de un 87\%). Por el contrario el confort térmico es el indicador más deficiente (sólo un $2 \%$ ) al igual que la producción de energías renovables, lo que es normal pues en el campus es actualmente inexistente.
Y finalmente podemos observar que el perfil del edificio de docencia es el que posee un mejor cumplimiento, 4 de los 7 indicadores han obtenido un cumplimiento superior al $80 \%$.

La Tabla 4 muestra los valores utilizados en el modelo de agregación, incluyendo los valores de interpretación $\left(Z_{i}\right)$ (resumidos en la Figura 6) y los resultados del nivel de apreciación $\left(w_{i}\right)$.

De acuerdo al nivel de apreciación que los tres tipos de usuarios (estudiantes, profesores-investigadores, administrativos) le entregaron a cada indicador, se observa que el consumo energético (en promedio de 30\%) es el indicador más importante y en segundo lugar el confort térmico (23\%), y por el contrario el indicador menos importante fue el olfativo (1\%).

Por otra parte, la Tabla 5 resume los resultados que se pueden obtener en los cuatro grados de compromiso, definidos en las ecuaciones [5] [6] [7] y [8], el peor $\left(\mathrm{s}_{\text {mín }}\right)$, el mejor $\left(\mathrm{s}_{\text {máx }}\right)$ cumplimiento, así como los dos casos intermedios $(\mathrm{s} \rightarrow 0)$ y $(\mathrm{s} \rightarrow 1)$.

En el cálculo de agregación (obtención del $I G A$ ), hemos decidido mostrar sólo los resultados que le dan a todos los indicadores el mismo peso de importancia (columna $s \rightarrow 1$ ), lo que se traduce en la media aritmética ponderada de los $Z_{i}$ por su nivel de apreciación $\left(w_{i}\right)$ [7].

Finalmente, la Figura 7 (representación gráfica de los valores IGA) muestra los resultados de los perfiles de aceptabilidad de los tres escenarios en los tres edificios estudiados. En los perfiles observamos que para los tres edificios el escenario económico es el más deficiente (en promedio de o,13), luego viene el escenario social (en promedio 0,25). Por otro lado el escenario medioambiental está mejor evaluado en el edificio de investigación y peor en el edificio de docencia.

Tabla 3. Los valores de las variables de observación $\left(y_{i}\right)$ y de las variables de interpretación $\left(z_{i}\right)$ de cada criterio.

\begin{tabular}{|c|c|c|c|c|c|c|c|}
\hline \multirow[t]{2}{*}{ CRITERIO } & \multirow{2}{*}{$\begin{array}{c}\text { INTERVALO } \\
] y_{\mathrm{o}}-y_{1}[ \\
F O\end{array}$} & \multicolumn{2}{|c|}{$\begin{array}{c}\text { Edificio de } \\
\text { Investigación }\end{array}$} & \multicolumn{2}{|c|}{$\begin{array}{c}\text { Edificio de } \\
\text { Administración }\end{array}$} & \multicolumn{2}{|c|}{$\begin{array}{c}\text { Edificio de } \\
\text { Docencia }\end{array}$} \\
\hline & & $\boldsymbol{y}_{i}$ & $z_{i}$ & $\boldsymbol{y}_{i}$ & $z_{i}$ & $\boldsymbol{y}_{i}$ & $z_{i}$ \\
\hline $\begin{array}{l}\text { Consumo de gas y electricidad } \\
\left(\mathrm{kWh} \mathrm{EF}_{\mathrm{EF}} \text { anuales } / \mathrm{m}^{2}\right)\end{array}$ & $\begin{array}{l}\text { ]50 - 300 }[ \\
\text { minimizar }\end{array}$ & 271 & 0,10 & 246 & 0,29 & 91 & 0,97 \\
\hline $\begin{array}{l}\text { Producción de energías renovables } \\
\left(\mathrm{kWh} \mathrm{EF}_{\mathrm{EF}} \text { anuales } / \mathrm{m}^{2}\right)\end{array}$ & $\begin{array}{c}{[0-50[} \\
\text { maximizar }\end{array}$ & $\mathrm{O}$ & 0,01 & $\mathrm{O}$ & 0,01 & $\mathrm{O}$ & 0,01 \\
\hline $\begin{array}{l}\text { Cantidad de emisiones de } \mathrm{CO} 2 \\
\left(\mathrm{~kg} . e q \mathrm{CO}_{2} \text { anuales } / \mathrm{m}^{2}\right)\end{array}$ & $\begin{array}{l}\text { ]5-145[ } \\
\text { minimizar }\end{array}$ & 74 & 0,81 & 69 & 0,85 & 24 & 0,98 \\
\hline Satisfacción olfativa \% & $\begin{array}{l}{[0-100]} \\
\text { maximizar }\end{array}$ & 50 & 0,81 & 50 & 0,81 & 50 & 0,81 \\
\hline Cantidad de vistas \% & $\begin{array}{l}\text { [o-5o[ } \\
\text { delimitar }\end{array}$ & 12 & 0,98 & 6 & 0,27 & 24 & 0,99 \\
\hline Distancia (máx) a las vistas (m) & $\begin{array}{l}\text { [o - 6[ } \\
\text { delimitar }\end{array}$ & 6,1 & 0,66 & 4,5 & 0,85 & 6,6 & 0,56 \\
\hline Cantidad de iluminación (lux) & $\begin{array}{l}\text { ]250 - 2500[ } \\
\text { delimitar }\end{array}$ & 314 & 0,01 & 554 & 0,48 & 810 & 0,99 \\
\hline Nivel de ruido $(\mathrm{dB}(\mathrm{A}))$ & $\begin{array}{l}\text { ]32-50[ } \\
\text { delimitar }\end{array}$ & 54 & 0,96 & 37 & 0,84 & 38 & 0,96 \\
\hline Temperatura interior $\left({ }^{\circ} \mathrm{C}\right)$ & $\begin{array}{l}\text { ]17-25[ } \\
\text { delimitar }\end{array}$ & 21 & 0,99 & 22 & 0,99 & 21 & 0,99 \\
\hline Velocidad del aire interior $(\mathrm{m} / \mathrm{s})$ & $\begin{array}{l}\text { ]o,15-o,25[ } \\
\quad \text { delimitar }\end{array}$ & 0,23 & 0,03 & $\mathrm{O}$ & 0,01 & $\mathrm{O}$ & 0,01 \\
\hline Humedad relativa (\%) & $\begin{array}{l}\text { ]40 - 6o[ } \\
\text { delimitar }\end{array}$ & 55 & 0,99 & 56 & 0,92 & 53 & 0,99 \\
\hline Satisfacción térmica (\%) & $\begin{array}{l}{[0-100]} \\
\text { maximizar }\end{array}$ & 18 & 0,22 & 50 & 0,81 & 50 & 0,81 \\
\hline
\end{tabular}




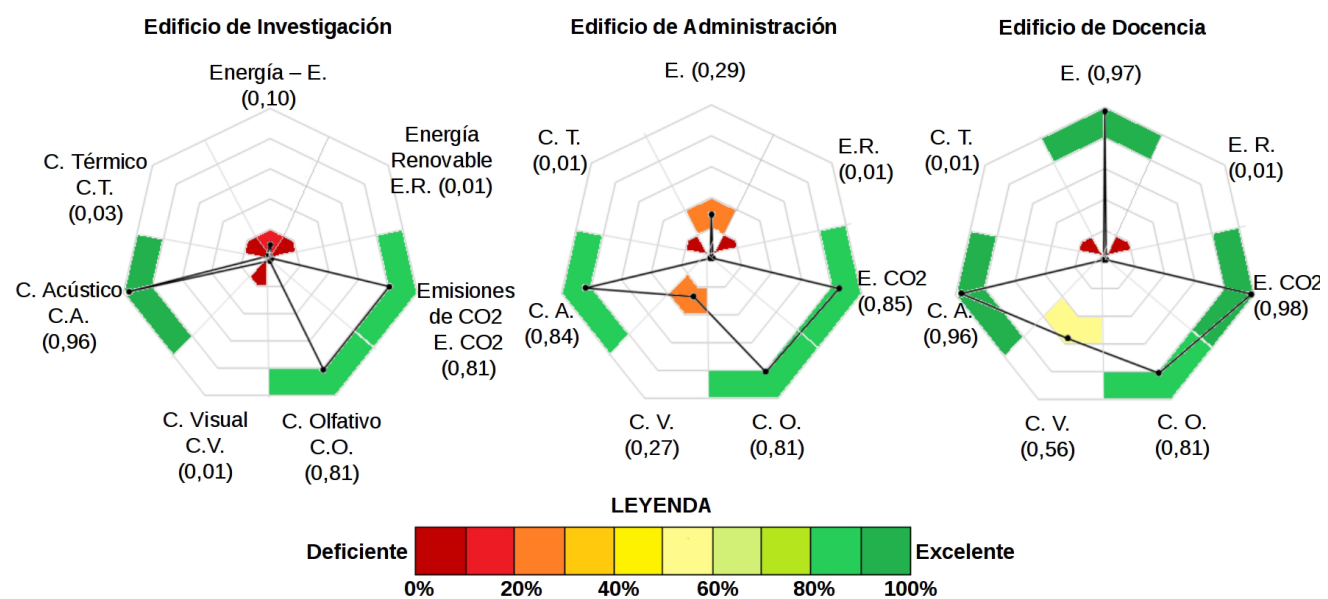

Figura 6. Perfiles de aceptabilidad $\left(Z_{i}\right)$ de los indicadores de los tres edificios estudiados.
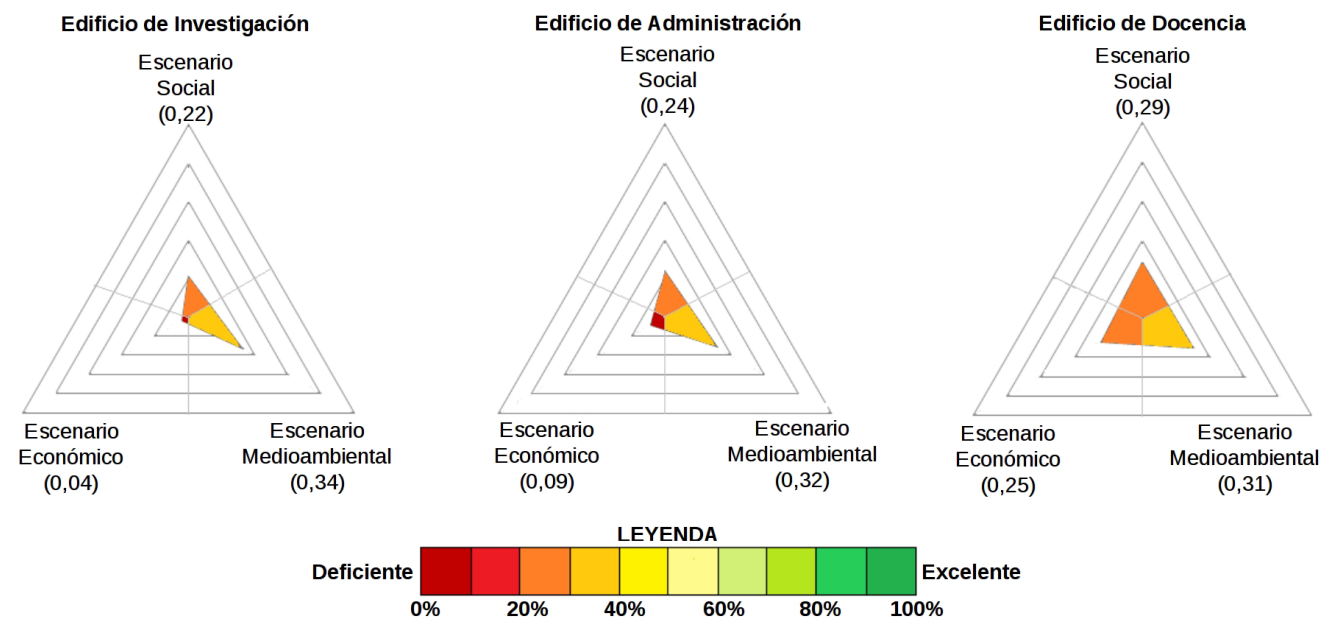

Figura 7. Perfiles de aceptabilidad (IGA) de los escenarios de los tres edificios.

Tabla 4. Los valores utilizados para la construcción de cada índice de aceptabilidad (IGA).

\begin{tabular}{|l|l|c|c|c|c|c|c|}
\hline \multirow{2}{*}{ ESCENARIO } & \multicolumn{2}{|c|}{ INDICADOR } & \multicolumn{2}{c|}{$\begin{array}{c}\text { Edificio de } \\
\text { Investigación }\end{array}$} & \multicolumn{2}{c|}{$\begin{array}{c}\text { Edificio de } \\
\text { Administración }\end{array}$} & \multicolumn{2}{c|}{$\begin{array}{c}\text { Edificio de } \\
\text { Docencia }\end{array}$} \\
\cline { 3 - 9 } & & $\boldsymbol{Z}_{\boldsymbol{i}}$ & $\boldsymbol{w}_{\boldsymbol{i}}$ & $\boldsymbol{Z}_{\boldsymbol{i}}$ & $\boldsymbol{w}_{\boldsymbol{i}}$ & $\boldsymbol{Z}_{\boldsymbol{i}}$ & $\boldsymbol{w}_{\boldsymbol{i}}$ \\
\hline \multirow{2}{*}{ Económico } & Consumo Energético & 0,10 & 0,35 & 0,29 & 0,30 & 0,97 & 0,26 \\
\hline \multirow{3}{*}{ Medioambiental } & Energías Renovables & 0,01 & 0,13 & 0,01 & 0,19 & 0,01 & 0,25 \\
\cline { 2 - 9 } & Emisiones de CO $\mathrm{CO}_{2}$ & 0,81 & 0,09 & 0,85 & 0,11 & 0,98 & 0,11 \\
\hline \multirow{3}{*}{ Social } & Confort Olfativo & 0,81 & 0,01 & 0,81 & 0,01 & 0,81 & 0,01 \\
\cline { 2 - 9 } & Confort Visual & 0,01 & 0,10 & 0,27 & 0,10 & 0,56 & 0,09 \\
\cline { 2 - 9 } & Confort Acústico & 0,96 & 0,08 & 0,84 & 0,07 & 0,96 & 0,05 \\
\cline { 2 - 9 } & Confort Térmico & 0,03 & 0,24 & 0,01 & 0,22 & 0,01 & 0,23 \\
\hline
\end{tabular}

Tabla 5. Los valores de cada escenario de decisión (IGA).

\begin{tabular}{|c|c|c|c|c|c|c|c|c|c|c|c|c|}
\hline \multirow{2}{*}{ ESCENARIO } & \multicolumn{4}{|c|}{ Edificio de Investigación } & \multicolumn{4}{|c|}{ Edificio de Administración } & \multicolumn{4}{|c|}{ Edificio de Docencia } \\
\hline & $\mathbf{s}_{\min }$ & $\mathbf{S} \rightarrow \mathbf{O}$ & $\mathbf{S} \rightarrow \mathbf{1}$ & $\mathbf{S}_{\text {máx }}$ & $\mathbf{s}_{\min }$ & $\mathbf{S} \rightarrow \mathbf{O}$ & $\mathbf{s} \rightarrow \mathbf{1}$ & $\mathbf{S}_{\text {máx }}$ & $\mathbf{s}_{\min }$ & $\mathbf{S} \rightarrow \mathbf{O}$ & $\mathbf{S} \rightarrow \mathbf{1}$ & $\mathbf{s}_{\text {máx }}$ \\
\hline Económico & 0,10 & 0,45 & 0,04 & 0,10 & 0,29 & 0,69 & 0,09 & 0,29 & 0,97 & 0,99 & 0,25 & 0,26 \\
\hline Medioambiental & 0,01 & 0,54 & 0,34 & 0,81 & 0,01 & 0,41 & 0,32 & 0,85 & 0,01 & 0,32 & 0,31 & 0,25 \\
\hline Social & 0,01 & 0,27 & 0,22 & 0,96 & 0,01 & 0,31 & 0,24 & 0,84 & 0,01 & 0,33 & 0,29 & 0,23 \\
\hline
\end{tabular}


Tabla 6. Los valores utilizados para la construcción del índice final de evaluación (IFE).

\begin{tabular}{|c|c|c|c|c|c|c|}
\hline \multirow{2}{*}{ ESCENARIO } & \multicolumn{2}{|c|}{$\begin{array}{c}\text { Edificio de } \\
\text { Investigación }\end{array}$} & \multicolumn{2}{|c|}{$\begin{array}{c}\text { Edificio de } \\
\text { Administración }\end{array}$} & \multicolumn{2}{|c|}{$\begin{array}{c}\text { Edificio de } \\
\text { Docencia }\end{array}$} \\
\hline & IGA & $\boldsymbol{w}_{i}^{\prime}$ & IGA & $w_{i}^{\prime}$ & IGA & $w_{i}^{\prime}$ \\
\hline Económico & 0,04 & 0,35 & 0,09 & 0,30 & 0,25 & 0,26 \\
\hline Medioambiental & 0,34 & 0,23 & 0,32 & 0,30 & 0,31 & 0,36 \\
\hline Social & 0,22 & 0,43 & 0,24 & 0,40 & 0,29 & 0,38 \\
\hline
\end{tabular}

Tabla 7. Los valores de cada escenario de decisión (IFE).

\begin{tabular}{|c|c|c|c|c|c|c|c|c|c|c|c|c|}
\hline \multirow{3}{*}{ IFE } & \multicolumn{4}{|c|}{ Edificio de Investigación } & \multicolumn{4}{|c|}{ Edificio de Administración } & \multicolumn{4}{|c|}{ Edificio de Docencia } \\
\hline & $\mathbf{s}_{\text {mín }}^{\prime}$ & $\mathbf{s}^{\prime} \rightarrow \mathbf{O}$ & $\mathbf{s}^{\prime} \rightarrow \mathbf{1}$ & $\mathbf{s}_{\text {máx }}^{\prime}$ & $\mathbf{s}_{\text {min }}^{\prime}$ & $\mathbf{s}^{\prime} \rightarrow \mathbf{o}$ & $s^{\prime} \rightarrow \mathbf{1}$ & $\mathbf{s}_{\text {máx }}^{\prime}$ & $\mathbf{s}_{\text {mín }}^{\prime}$ & $\mathbf{s}^{\prime} \rightarrow \mathbf{o}$ & $\mathbf{s}^{\prime} \rightarrow \mathbf{1}$ & $\mathbf{s}_{\text {máx }}^{\prime}$ \\
\hline & 0,04 & 0,13 & 0,19 & 0,34 & 0,09 & 0,19 & 0,22 & 0,32 & 0,25 & 0,29 & 0,29 & 0,31 \\
\hline
\end{tabular}

La Tabla 6 muestra los valores de los IGAs (resumidos en la Figura 7) y los valores de apreciación ( $w_{i}^{\prime}$ ) utilizados en la segunda familia de agregación, que nos han servido para la determinación del índice final de evaluación.

Por otro lado, en función del nivel de apreciación que los usuarios de los edificios le entregan a los diferentes escenarios, podemos observar que en general los escenarios económico y social reciben la misma importancia en el edificio de administración, mientras que el escenario social del edificio de investigación se muestra un poco más elevado con un $43 \%$.

$\mathrm{Al}$ igual como en el caso anterior nuevamente se presenta en la Tabla 7 los resultados que se pueden obtener en los cuatro grado de compromiso del IFE para esta segunda familia de agregación, el peor ( $\mathrm{s}_{\text {mín }}$ ), el mejor cumplimiento $\left(\mathrm{s}_{\text {máx }}\right)$ y los dos casos intermedios (ecuaciones [5] [6] [7] y [8]).

Los valores de s' $\rightarrow 0$ para la media geométrica y s' $\rightarrow 1$ para la media aritmética son muy similares en medida, debido a que cada nivel de apreciación de cada escenario es semejante en los tres edificios. Al igual que en el caso anterior, hemos elegido mostrar solamente los resultados obtenidos de la media aritmética ponderada de los indicadores, para así demostrar de manera más fidedigna las apreciaciones de los entrevistados.

Es así como el índice final de evaluación para el edificio de investigación es igual a 0,19, lo que indica que en un escenario pesimista el edificio sólo cumple un 19\% de aceptación, en el edificio de administración es igual a 0,22 y en el edificio de docencia es igual a 0,29, siendo el edificio mejor evaluado de los tres.

\section{DISCUSIÓN Y CONCLUSIONES}

Uno de los principales requerimientos de un indicador de evaluación es que su resultado debe ser comprensible y adaptable en diferentes contextos. Con la utilización del método de optimización, hemos podido simplificar la comparación e inclusión de los indicadores en la metodología de ayuda à la decisión a través de la función de aceptabilidad $\left(Z_{i}\left({ }_{y}\right)\right)$. Por otra parte esto da cabida a la adaptación de la metodología a diferentes contextos o países con una correcta elección y construcción de los intervalos de sus indicadores.

La metodología presentada ha permitido mostrar y reagrupar todos los resultados a diferentes niveles de evaluación: sólo a nivel de indicadores, por cada escenario de decisión (IGA) o como una única evaluación final (IFE).
Además, la incorporación de la opinión de los usuarios permite, por cada edificio tipo, mostrar al gestor el grado de satisfacción que sienten los ocupantes de esos edificios quienes representan una pieza clave en la evaluación del comportamiento cotidiano de éstos.

En función a los resultados obtenidos de la aplicación de la metodología de evaluación en tres tipos de edificios de un campus universitario francés, se observó que:

- los indicadores con mejor resultado de cumplimiento (Figura 6) fueron el confort acústico, el confort olfativo y luego las emisiones de $\mathrm{CO}_{2}$, que se opone a los resultados de la apreciación que los usuarios les dieron.

- el escenario de decisión (IGA) (Figura 7) con mejor cumplimiento fue el medioambiental, pero a la vez fue un escenario «poco» importante para los usuarios, con una apreciación que alcanza en promedio sólo el 30\%.

Los siguientes pasos para la mejora de la metodología serán:

- Extender el caso piloto a otro tipo de campus universitario, por ejemplo uno de ciencias humanas, en donde la investigación no requiere de gran equipamiento tecnológico (caracterizado por aumentar la factura energética) en comparación al campus aquí estudiado

- Extender el estudio a otros contextos geográficos para validar la adaptación de los intervalos de los indicadores

- Incorporación de otros indicadores o tópicos

Los resultados obtenidos de la aplicación de la metodología deben ser complementados con la cuantificación económica del coste de cada decisión a tomar. Sin embargo aún en esta fase ya son una fuente de información objetiva del comportamiento del edificio lo cual le ha permitido al gestor del campus «Ciencias y Tecnologías» de la Universidad de Burdeos, en Francia, conocer la opinión de sus usuarios e incorporarla en las políticas energéticas de la Universidad, para comportamientos sostenibles y durables en el tiempo.

\section{AGRADECIMIENTOS}

Los autores agradecen a los interlocutores de la Dirección de Patrimonio Inmobiliario de la Universidad Burdeos por la ayuda en las distintas campañas de medidas. Y al programa Becas Chile de CONICYT por el financiamiento para la realización de este estudio. 


\section{REFERENCIAS}

(1) Kajikawa, Y., Inoue, T., Goh, T. (2011). Analysis of building environment assessment frameworks and their implications for sustainability indicators. Sustainability Science, 6(2): 233-246, doi: http://dx.doi.org/10.1007/s11625-011-0131-7.

(2) OECD. (1993). OECD Core set of indicators for environmental performance reviews. Paris, France: Organisation for Economic Cooperation and Development (OECD).

(3) Schulze, I., Colby, M. (1995). A Conceptual Framework to Support the Development and Use of Environmental Information for Decision-Making. USA: Environmental Statistics and Information Division, Office of Policy, Planning and Evaluation - EPA (Agencia de Protección Ambiental de Estados Unidos).

(4) International Institute for Sustainable development (IISD). (2010). Compendium of Sustainability Indicators. http:// www.iisd.org/measure/compendium/searchinitiatives.aspx.

(5) Parris, T. M., Kates, R. W. (2003) Characterizing a sustainability transition: Goals, targets, trends, and driving forces. Proceedings of the National Academy of Sciences, 100(14): 8068-8073, doi: http://dx.doi.org/10.1073/pnas.1231336100.

(6) Macías, M., García-Navarro, J. (2010). Metodología y herramienta VERDE para la evaluación de la sostenibilidad en edificios. Informes de la Construcción, 62(517): 87-100, doi: http://dx.doi.org/10.3989/ic.08.056.

(7) Ding, G. K. C. (2008). Sustainable construction-The role of environmental assessment tools. Journal of Environmental Management, 86(3): 451-464, doi: http://dx.doi.org/10.1016/j.jenvman.2006.12.025.

(8) Crawley, D., Aho, I. (1999). Building environmental assessment methods: applications and development trends. Building Research \& Information, 27(4-5): 300-308, doi: http://dx.doi.org/10.1080/o96132199369417.

(9) Lecuona-Neumann, A., Izquierdo-Millán, M., Rodríguez-Aumente, P. (2005). Investigación e impacto ambiental de los edificios. La Energía. Informes de la Construcción, 57(498): 47-61, doi: http://dx.doi.org/10.3989/ic.2005.v57.i498.477.

(10) Tendero, R., García-de Viedma, M. (2011). Edificación para un desarrollo sostenible: una actividad modal. Informes de la Construcción, 63(521): 75-87, doi: http://dx.doi.org/10.3989/ic.09.034.

(11) Valderrama-Ulloa, C., Cohen, A., Lagière, P., Puiggali, J.R. (2011). Análisis del comportamiento energético en un conjunto de edificios multifuncionales. Caso de estudio: un campus universitario. Revista de la Construcción, 10(2): 26-39, doi: http://dx.doi.org/10.4067/So718-915X2011000200004.

(12) Ulloa, C., Crepin, J., Lagière, P., Puiggali J.R. (2012). Adaptation of environmental indicators to assess the existing buildings to different requirements of users. En 1st International Conference on Building Sustainability Assessment, (pp. 375-385). Porto, Portugal.

(13) Saaty, T. L. (1990). How to make a decision: the analytic hierarchy process. European journal of operational research, 48(1): 9-26, doi: http://dx.doi.org/10.1016/0377-2217(90)90057-I.

(14) Martínez, E., Álvarez, M., Arquero, Á., Romero, M. (2010). Apoyo a la selección de emplazamientos óptimos de edificios. Localización de un edificio universitario mediante el Proceso Analítico Jerárquico (AHP). Informes de la Construcción, 62(519): 36-45, doi: http://dx.doi.org/10.3989/ic.o8.052.

(15) Harrington, E. C. (1965). The Desirability Function. Industrial Quality Control, 21(10): 494-498.

(16) Derringer, G., Suich, R. (1980). Simultaneous-optimization of several response variables. Journal of Quality Technology, 12(4): 214-219.

(17) Sebastian, P., Quirante, T., Ho Kon Tiat, V., Ledoux, Y. (2010). Multi-objective optimization of the design of two-stage flash evaporators: Part 2. Multi-objective optimization. International Journal of Thermal Sciences, 49(12): 2459-2466, doi: http://dx.doi.org/10.1016/j.ijthermalsci.2010.07.002.

(18) Wagner, T., Trautmann, H. (2010). Integration of Preferences in Hypervolume-Based Multiobjective Evolutionary Algorithms by Means of Desirability Functions. IEEE Transactions on Evolutionary Computation, 14(5): 688-701, doi: http://dx.doi.org/10.1109/TEVC.2010.2058119.

(19) Scott, M. J., Antonsson, E. K. (1998). Aggregation functions for engineering design trade-offs. Fuzzy Sets and Systems, 99(3): 253-264, doi: http://dx.doi.org/10.1016/So165-0114(97)00032-8.

(20) MEEDDM. (2010). Caractéristiques thermiques et aux exigences de performance énergétique de bâtiments. Francia : Ministére de l'écologie, de l'énergie, du développement durable et de la mer, en charge des technologies vertes et des négociations sur le climat (MEEDDM).

(21) Le Moniteur. (2007). Tertiaire neuf: quelles différences entre Effinergie, Minergie et Passivhaus?. http://www.lemoniteur. fr/201-management/article/etudes-de-cas/684957-tertiaire-neuf-quelles-differences-entre-effinergie-minergie-et-passivhaus.

(22) Mandallena, C., Lagière, P., Puiggali, J.R. (2008). Evaluation et amélioration des performances environnementales d'un bâtiment tertiaire en exploitation. European journal of environmental and civil engineering, 12(4): 333-346. doi: http://dx.doi.org/10.1080/19648189.2008.9693017.

(23) Ganslandt, R., Hofmann, H. (1992) Handbook of lighting design. Alemania: ERCO.

(24) Caisse des Depôts, Conférence des présidents d'université. (2009). Cartographie Energie- $\mathrm{CO}_{2}$ du patrimoine universitaire français. http://www.cartoco2campus.com/.

(25) ASHRAE. (2004). ASHRAE Standard 55 - 2004 for High Performance Building - Thermal environmental conditions for human occupancy. Estados Unidos : The American Society of Heating, Refrigeration and Air-conditioning Engineers (ASHRAE).

(26) CSST. (2004). Guide: Confort thermique à l'intérieur d'un établissement. Canada: Commission de la Santé et de la sécurité du travail du Québec (CSST).

(27) Perdrix, A., Parat, S., Liaudy, S., Maitre, A. (2005). Syndrome des bâtiments malsains (SBM). Revue francophone des laboratoires, (373): 67-72, doi: http://dx.doi.org/10.1016/So338-9898(05)80237-9.

(28) von Schirnding, Y. (2002). Health in Sustainable Development Planning: The role of indicators. World Health Organization (WHO). 